\title{
THE EFFECTS OF WEATHERING ON MECHANICAL PROPERTIES OF GLASS FIBER REINFORCED PLASTICS (GRP) MATERIALS.
}

\author{
H. Abdullah \\ Department of Mechanical Engineering, University of Guelph, School of Engineering, Guelph, Ontario, \\ Canada N1G 2Wl. \\ habdulla@uoguelph.ca

\section{S. Al Araimi and R. A. Siddiqui} \\ Department of Mechanical and Industrial Engineering, Sultan Qaboos University, P.O.BOX33, Al-Khod, \\ Muscat-123, Sultanate of Oman \\ alaraimi@squ.edu.om,siddiqui@squ.edu.om
}

\begin{abstract}
Glass fiber reinforced plastics composite is extensively used as a structural material for pools, oil pipes and tanks because it has good corrosion resistance properties. The effects of weathering on the mechanical properties of glass fiber reinforced plastics (GRP) in the Sultanate of Oman have been studied. The tensile and three point bend specimens were exposed to outdoor conditions (open atmosphere) in sunlight and tested for various intervals of time. It was observed that as the exposure time to sunlight, ultraviolet radiation and dust increases the mechanical properties of GRP materials decrease. The effects of relative humidity $(\% \mathrm{RH})$ on the mechanical properties were also studied. It was found that as the relative humidity increased in the atmosphere during the exposure time, the tensile strength, flexural strength and modulus of elasticity are lowered. This work has revealed that the decrease in the mechanical properties of GRP under weathering conditions is subjected to atmospheric conditions such as humidity, temperature, ultraviolet radiation and pollutant.
\end{abstract}

Key Words: Weathering, Glass-Fiber Reinforced Plastics, Degradation

\section{INTRODUCTION}

GRP is an industrial term that denotes one of the composite materials, which is called glass fibre reinforced plastics. The GRP is used to control corrosion problems in oil fields, marine applications, chemicals and industrial plants. The successful performances of fibre glass reinforced thermo setting plastics have led to its wide use in corrosive services compared to other metallic materials. Also, due to its improved properties such as high specific strength and stiffness to weight ratio, glass fibre reinforced polymers composites are utilised successfully as structural material. The mechanical properties of fibreglass reinforced composite material depend on its composition, namely the fibre and the matrix plus the quality of the fibre matrix interface. The percentage of glass fibre (by volume) in reinforced plastics usually ranges between $30 \%$ to $60 \%{ }^{[1]}$. The first use of GRP material by TOTAL took place in 1975 at one of the offshore oil fields in the Middle East ${ }^{[1]}$. The objective was to control the corrosion of firewater and cooling water systems by replacing the existing galvanised carbon steel pipes material with GRP.

Most of the literature available on the degradation is related to the polymers, while studies related to the effects of weathering or environmental resistance on GRP itself are scarce.

The environmental effects on the material integrity of fibre reinforced polymer matrix composites (PMCs) was investigated by Dispennette et al. ${ }^{[2]}$. The composite materials were composed of carbon fibre reinforced with epoxy matrix. They were exposed to selected environments i.e. moisture, gamma radiation and atomic oxygen (AO) to induce varying degree of degradation. The studied properties were specifically glass transition temperature (Tg), weight loss and modulus. From this investigation, it was determined that the erosion behaviour of these materials was significantly effected by the environmental exposures. However, the properties like modulus, were slightly affected.

Ontsuka ${ }^{[3]}$ reported that the polymer degradation took different forms, such as fracturing, cracking, and chalking, depending on the nature of polymers. He showed that the degradation of polymers depends not only on the environment in which they are practically used, but also on their fabrication processes.

The prediction of the polymeric material service life when exposed to outdoor conditions was investigated by Tomiita ${ }^{[4]}$. The weather abilities of this material used outdoors have not been analysed in details. Therefore, it was essential to establish a service life prediction system of polymeric materials. The degradation factors include solar ultraviolet irradiation, heat and moisture attack on polymers exposed outdoors. The authors expressed the degradation phenomena by mathematical models as a function of degradation factors and elapsed time. They developed a computer model to determine degradation material constants and predict property changes over time by simulating degradation. 
Al-Bastaki and Al-Madani ${ }^{[5]}$ exposed the GRP specimens directly to solar radiation and natural atmospheric conditions in Bahrain. Generally, the weather in Bahrain has high temperature and humidity during the summer and very little rain during the winter. The flexural strength of the specimens exposed to outdoor conditions showed a little variation. The tensile specimens had a reduction in failure strain and an increase in the modulus of elasticity. The environmental effects on polyesters laminates reinforced with chopped strand glass fibre mat were also investigated by Abraham ${ }^{[6]}$ in marine environment. The experimental results have shown a slow decrease in mechanical properties of GRP with an increase in the ageing time.

Bonnian and Bunsell ${ }^{[7]}$ work on epoxy resin have revealed that degradation usually occurs in environments with concentration of water. Schutte ${ }^{[8]}$ studied the environmental attacks by moisture on the durability of glass fiber polymer composites. It has been found that moisture could degrade the strength of the glass fiber, plasticizer and swell or micro-crack the resin. Also, the fiber matrix interface could be weakened by either chemical or mechanical attack. The relative rates of these degradation processes were function of the chemistry of the resin, temperature, length of time of exposure, degree of stress (whether cyclic or static), chemistry and morphology of coating of coupling agent on the glass fiber, and type of glass fibre.

Bunsell ${ }^{[9]}$ studied the influence of water absorption and heat on the behaviour of epoxy resins and glass fibre. The study revealed that the degradation usually occurred in environment with concentration of water greater than 70 to $80 \%$ R.H. The degradation effects were accelerated particularly above $70^{\circ} \mathrm{C}$. Degradation occurred by hydrolysis, by resin swelling and by cracking. Meanwhile, glass fibres resisted quite well to the attack of neutral water, but were damaged when contacted with acid or alkaline environments.

The effects of saline treated fibre on impact and tensile properties of hydrothermal aged glass fabric/epoxy laminates were examined by Morii et al [10]. The specimens were immersed in hot distilled water. The static tensile,the impact flexural and change in weight were measured. The weight loss occurred for methacryl treated laminate as a result of debonding of fibre/matrix at the interface and the water penetrates into this interface. This degradation of interface affected the tensile and impact properties and it induced the greater modulus and strength reduction.

Castaing and Lemoine ${ }^{[1]}$ evaluated the mechanical characteristics versus time to investigate the effects of water absorption and osmotic degradation on long-term behavior of glass fiber reinforced plastics. It was shown that the main effects of water on the composite mechanical properties are the plasticization of the matrix and the osmotic delaminating of plies, causing a decrease in mechanical properties. On the other hand, gelcoat blistering had no influence on the durability of plastics.

Davies et al ${ }^{[12]}$ investigated the influence of water absorption on tensile properties and shear fracture toughness of glass/polypropylene. The immersion time was up to 5 months at 50 degree ${ }^{\circ} \mathrm{C}$. It was observed that the absorption of distilled water was more than seawater. A very small change in the transverse tensile modulus was observed after immersion in the water, but fracture toughness decreases significantly. The reduction in fracture toughness is attributed to the degradation of the matrix due to water absorption as observed by the scanning electron microscope.

A study on photodegradation and photo stabilisation of polymer was conducted in 1940s, when it was observed that degradation in air also involved simultaneous oxidation. The basic mechanism of photooxidation degradation was established by Bolland and $\mathrm{Gee}^{[13]}$. An investigation of accelerated weathering and photo degradation in polymer was conducted by Kampf et al. ${ }^{[14]}$. The work summarised knowledge of the primary processes of photodegradation, emphasising on the significance of incoming light wavelength. The paper highlighted the significance of the wavelength of incoming light. The work also discussed various methods of photodegradation.

The effects of the Low Earth Orbital (LEO) atomic oxygen and ultraviolet radiation on the degradation of polymers were presented by Dever ${ }^{[15]}$. The ultraviolet radiation and the environmental atomic oxygen can alter the chemistry of polymers resulting in degradation. The author introduced a survey of the interaction and possible damage mechanisms for environmental atomic oxygen and ultraviolet radiation exposure of polymers commonly used in LEO.

From the literature survey presented above, it is obvious that most of previously published work was conducted on polymers or epoxy resin instead of fibre reinforced polymers. This paper is intended to investigate the effect of atmospheric conditions on the mechanical properties of GRP conducted under the most severe weather conditions in the Sultanate of Oman. The experimental work was carried out in Muscat, which is one of the hottest cities in the world. The rainfall is generally light and irregular but the average relative humidity during summer varies between 75$85 \%(\mathrm{RH} \%)$ and the temperature can exceed $50{ }^{\circ} \mathrm{C}$.

\section{EXPERIMENTAL PROCEDURE}

The glass fibre reinforced plastic (GRP) sheets were manufactured by Amiantit Oman Company (Muscat, Sultanate of Oman) by hand lay up process and consist of:

- Resin to BS3532/ Crystic 491 E Isopthalic, from Scott Bader, UK

- $\quad$ E-glass mat to BS 3496

- Resin to glass ratio 2.5:1

The tensile specimens are machined according to ASTM No. D 3039 and three point bend specimens are prepared according to ASTM D 790-91 standards. The length, width, thickness and weight of each specimen were measured and recorded. All the specimens were hanged on rack and were subjected to natural weathering, consist of changing in temperature, 
humidity, solar radiation as well as other factors (such as pollutants present in the environment). Weather conditions were recorded daily during the experimental period at Sultan Qaboos University Metrology Centre. The exposure time was little more than one year (389 days) in the city of Muscat, Sultanate of Oman. The general atmospheric conditions were high temperature, high humidity in summer and very little rain during winter. The samples were removed periodically during this exposure period (every 10 days on the average) and tested for tensile strength, three-point bend test and moisture content. The tensile test was conducted by Lloyd machine with a strain rate of $0.5 \mathrm{~mm} / \mathrm{min}$. The three point bend tests were carried out with Lloyd instrument with a speed of $2 \mathrm{~mm} / \mathrm{min}$. The flexural strength, the load and displacement data, modulus of elasticity and moisture content were calculated from graphs obtained from the testing machines.

\section{RESULTS AND DISCUSSION}

Plastics, rubber fibres, paints and lacquers are all degraded in sunlight by basically the same free radical mechanism. Similarly, prolonged exposure of GRP under natural weathering which includes moisture, temperature ultraviolet radiation etc. will result in degradation of the material. The mechanical properties of a composite depend on the properties of their components namely the fibres and matrix plus the quality of fibre matrix interface. It was difficult to have uniform composition of glass fiber and resin through out the material, especially, by hand lay up process. Therefor, the experimental results in Fig. 1 to 6 show scattering due to the non-uniform composition of GRP produced by hand lay up process.

The standard GRP specimens were tested after they were exposed to outdoor weathering. The effect on the GRP's tensile strength (Mpa $=145$ psi), after exposing in the natural environment for the period between 7 to 389 days, is presented in Fig. 1. Minimum of three specimens was tested at each interval and the average results were plotted as shown in Fig. 1. It is interesting to note that as the exposure time increases an over all slow decrease in tensile strength of GRP was observed.

Fig. 1 Effect of exposure time on the tensile strength of GRP
Fig. 2 Effect of exposure time on the weight of GRP

The change in the weight of the GRP (due to humidity) when exposed to the open atmosphere is shown in Fig. 2. It is obvious from the experimental results that for a prolonged exposure to humidity, the matrix of the composite material will absorb water, or free diffusion of water will take place in the resin without interaction with the material, Fig. 2. Also, it shows inconsistency in the results of weight gain by the specimen. This can be expressed as a loss in weight, which is due to evaporation of moisture from the specimens because of high temperature and low humidity during summer periods. The negative value on the graph represents a loss in weight (original weight) and the positive values means a gain in weight by the specimen exposed for a period of 389 days.

The effect of average relative humidity on the tensile strength of GRP is plotted in Fig. 3 respectively. It is found that as the relative humidity in the atmosphere exceeds beyond $40 \%$, the degradation increases. A decrease in tensile strength in most of the GRP specimens was observed with ageing time. The decrease in tensile strength could be due to swelling and shrinkage, which is caused by the entry of water via diffusion through the resin. This may break the bond between fibers and matrix leading to a fall in tensile strength of GRP.
Fig. 3 Effect of average relative humidity on the tensile strength of GRP 
Figure 4 shows the effect of exposure time in days on flexural strength of GRP. It is observed that as the exposure time increases, the flexural strength of the material decreases. This is because of the effect of ultraviolet radiation, moisture content and heat as shown in Figs. 1-4.
Fig. 4 Effect of exposure time on the flexural strength of GRP

The effect of average relative humidity $(\% \mathrm{RH})$ on the flexural strength of GRP under the outdoor conditions (ultraviolet radiation, moisture and sunlight) is plotted in Fig. 5. The results show that as the exposure time and the percentage relative humidity $(\% \mathrm{RH})$ increases in the atmosphere, the flexural strength of GRP decreases. The variation of modulus of elasticity (E) of GRP composite materials as a function of exposure time is shown in Fig. 6. A decrease in $\mathrm{E}$ is evident from the graphs when the material was exposed to sunlight radiation, humidity and temperature.

Fig. 5 Effect of average relative humidity on flexural strength of GRP

However, the following observations were recorded during the experimental work. The first one is the effect of a pollutant. It was observed that a thin layer of sand covers the specimens during its exposure in the atmosphere. This thin layer of sand absorbs significant amount of solar radiation and ultraviolet radiation and thus, affects the temperature of the specimens. The second observation was yellowing of the specimens when exposed to the outdoor conditions. Yellowing of matrix under the influence of the short wavelength of solar radiation is also a usual cause of degradation. The yellowing effect depends on the composition of the resin as well as on the type and addition of the ultraviolet stabiliser present in the resin. The yellowing is an important factor, which causes the degradation of the resin and an increase in the brittleness of the material. Hence, affecting the mechanical properties of the material.

Fig. 6 Effect of exposure time on the modulus of elasticity (E) of GRP.

There are numbers of mechanisms that contributed to the degradation processes of the tested specimens such as mismatch in the coefficients of thermal expansion between fibre and the matrix, swelling and shrinkage of resin during moisture absorption, hydrolysis and photooxidation, thermal decomposition and surface erosion. In addition, the absorption of water by resin causes a fall in glass transition temperature $(\mathrm{Tg})$.

The decrease in mechanical properties such as tensile strength, flexural strength and modulus of elasticity of GRP on prolong exposure to humidity can be justified on the following basis: in most environments, organic matrix composite will absorb water, and the presence of water may cause changes in the constituents. The simplest model of water up take is based on the free diffusion of water into the resin without interaction with the material ${ }^{[11]}$. The absorption of moisture gives rise to a reduction of $\mathrm{Tg}$ and may break the bonds between fibres and matrix, leading to reduction in load transfer and a fall in mechanical properties. The degradation by hydrolysis is caused by differential swelling between the fibres and the resin, which produces serious cracks in the composite material and consequently affect the mechanical properties.

Bolland and Gee established ${ }^{[13]}$ established the basic mechanism of photooxidation degradation. The mechanism involves an initiation reaction of free radical formation by chain scission or some other bond cleavage, which is in the case of outdoor exposure of GRP materials, is caused by the ultraviolet radiation of sunlight. This scission causes accelerated degradation of the polymer chain, therefore the mechanical properties 
of the tested GRP specimens decreases with the increase in the exposure time.

Accentuation is another sign of deterioration for both polymers and epoxy/glass. As weathering proceeds, the thin resin film above the fibre strand bulges until it eventually burst, the exposed fibre come to the surface and promote dirt pick up. Since the fiber are exposed directly to the atmosphere and there is difference between the thermal expansion of fiber and the matrix, the breaking of the bond between fiber and matrix will take place. As a result, the mechanical properties of the composite material will be decreased.

\section{CONCLUSION}

The following conclusions can be drawn from the results of the present investigation:

The decrease in the mechanical properties, under weathering conditions is subjected to atmosphere conditions such as humidity, temperature, ultraviolet radiation, and pollutant. In most environments, organic matrix composite absorbs water/moisture by diffusion and the presence of water/moisture causes changes in the constituents. Also, it affects the resin by swelling and shrinking, which can break the bonds between fibre and matrix, leading to a reduction in load transfer and fall in the mechanical properties of GRP. A similar effect between fiber and matrix is possible due to the difference in the thermal expansion of the materials.

Sunlight causes some degradation in the organic matrix of the GRP, it also involves simultaneous oxidation. This is due to the initiation reaction of forming free radical followed by chain scission cleavage. Scission accelerates degradation of the polymer chain hence the mechanical properties are effected.

Although, sunlight generally causes some degree of surface deterioration in the organic matrix of a composite. It has become clear from various investigations that the combined effect of temperature and humidity is the most aggressive environmental factor affecting GRP in outdoor exposure.

\section{REFERENCES}

[1] C. F. P. Aubert, "Use of GRP Materials in Piping Systems. The Experience of TOTAL," Materials Engineering Proceedings of International Conference on Off-Shore Mechanics and Arctic Engineering - OME V3, Pt A, 1993 Published by ASME, New York, NY, USA, pp. 279-283, 1993.

[2] J. M. Dispennette, L. Seng, B. Z. Jang, and R. C. Linton, "Environmental Effects on the Material Integrity of Fiber Reinforced Polymer Matrix Composite," 50 years of progress in Materials and Science Technology International SAMPE Technical Conference, Vol. 26, SAMPE, Covina, CA, USA, pp. 119-127, 1994.

[3] H. Ontsuka "Degradation of Polymers-Causes and Methods for Estimation," Zairyo to Kankyo/Corrosion Engineering, Vol. 43, No. 6, pp. 340-346, June 1994.

[4] T. Tomiita, "Service Life Prediction System of Polymeric Materials Exposed Outdoors," Construction and Building Materials, Vol. 8, No. 4, pp. 223-226, December 1994.

[5] N. M. S. Al-Bastaki, and H. M. N. Al-Madani, "Effect of Six Months Exposure to Atmospheric Conditions on the Mechanical Properties of GRP," Modelling, Measurement and Control, C: Energetics, Chemistry Earth, Environmental \& Bio-medical Problem, Vol. 49, No. 1-3, pp. 22-28, 1995.

[6] T. Abraham, "Environmental Effect on Polyester Laminates Reinforced with Chopped Strand Glass Fibre Mats," Kautschuk und Gummi Kunststoffe, Vol. 49, No. 5, 350-353, May 1996

[7] P. Bonnian and A. R. Bunsell " A Comparative Study of Water Absorption Theories Applied to Glass Epoxy Composites, “ Journal of Composite Materials Vol. 15, pp. 272-278, 1981.

[8] C. L. Schutte, "Environmental Durability of Glass Fiber Composites," Materials Science and Engineering: R: Reports Vol. 13, No. 7, pp. 265-324, November 1994.

[9] A. R. Bunsell, "Hydrothermal Ageing of Composite Materials," Revue de 1' Institut Francais du Petrole, Vol. 50, No. 1, pp. 61-67, Jan.-Feb. 1995.

[10] T. Morii, H. Hamada N. Ikuta, M. Desaeger, and I. Verpoest, "Effect of Saline Treated Fiber on Impact Properties of Hydrothermal aged Glass Fabric/Epoxy Laminates," SAMPE Technical Conference, Vol. 27, pp. 1048-1058, 1995.

[11] P. Castaing, and L. Lemoine, "Effect of Water Absorption and Osmotic Degradation on Long-Term Behaviour of Glass Fiber Reinforced Polyester," Polymer Composites, Vol. 16, No. 5, pp. 349-356, October 1995.

[12] P. Davies, F. Pomies, and L. A. Carlsson, "Influence of Water Absorption on Transverse Tensile Properties and Shear Fracture Toughness of Glass/Polypropylene," Journal of Composite Materials, Vol. 30, No. 9, pp. 1006-1019, 1996.

[13] J. L Bolland and G. Gee, Transactions Faraday Society, Vol. 42, pp. 236-244, 1946.

[14] G. Kampf, K. Sommer, and E. Zirngiebl, "Studies in Accelerated Weathering Part I- Determination of the Activation Spectrum of Photodegradation in Polymers," Progress in Organic Coatings, Vol. 19, No. 1, pp. 69-77, April 1991

[15] J. A. Dever, "Low Earth Orbital Atomic Oxygen and Ultraviolet Radiation Effects on Polymers," FlightVehicle Materials, Structures and Dynamics- Assessment and Future Directions, Vol. 2, pp. 422-433, 1994.

\section{BIOGRAPHY}

Hussein A Abdullah received his Ph.D Degree from the University of Glasgow in 1992. He has been a researcher at the Department of Mechanical Engineering, University of Glasgow. He joined Incorporated Technology (ITL) as consultant in 1991. In 1995 he joined the Sultan Qaboos University as Assistant Professor, in the Department of Mechanical \& Industrial Engineering. At present he is working as Associate Professor at the school of Engineering, University of Guelph Canada. His major research interests are manufacturing systems, CAD /CAM materials processing, robotics, and mechatronic systems. 
Saeed Ali Al-Araimi received his $\mathrm{PhD}$ degree from the University of Missouri-Rolla in 1993. Prior to joining SQU, he worked as Deputy Director General of Industry in the Ministry of Commerce \& Industry in Sultanate of Oman. He received his B.Sc. (Cum Laude) in Industrial Engineering from University of Haven and MEM from Northwestern University. His current research interest includes Multi-Criteria Decision-Making, Total Quality Management, Project Management, Industrial Management and Engineering Education.
Rafiq Ahmad Siddiqui completed his B.Sc (Hons) and M.Sc degree in Chemical Engineering from the University of Punjab. Later in 1966 he received his Diplom in Metallurgy from W. Germany \& $\mathrm{PhD}$ in Metallurgy from University of Strathclyde U.K in 1974. $\mathrm{He}$ has worked in various Universities of Pakistan, Canada, Saudi Arabia, \& Sultanate of Oman as Assistant Professor, \& Associate Professor. He joined the Sultan Qaboos University, Department of Mechanical Engineering in 1988. He has been involved in teaching different courses in Materials Science as well as in Manufacturing Processing. His major interest is in the field of Metallography \& Microstructure, Failure Analysis \& Preventions, Fractro-graphy Corrosion \& Composites Materials. 